\title{
GET OUT INSIDE: PROGRAMMING TOYS 2.0 TO ESCAPE THE ISLAND
}

\author{
Elisabete Cunha ${ }^{1,3}$, Isabel Cabrita ${ }^{2}$ and Lina Fonseca ${ }^{1}$ \\ 1 Polytechnic Institute of Viana do Castelo, Portugal, ${ }^{2}$ CIDTFF- Research Centre on Didactics and \\ Technology in the Education of Trainers, University of Aveiro, Portugal, ${ }^{3}$ ARC4DigiT
}

\begin{abstract}
Escaping from an island is the context used to promote the resolution of geometric problems that involve the programming of toys 2.0 in a collaborative environment. In this article, we characterize the signs produced in this experience considering the Theory of Semiotic Mediation and we analyse its impact on learning from the student's perspective. Thus, artifact signs, pivot signs and mathematical signs were cyclically mobilized and in different directions depending on the nature of the challenge. Of the students surveyed in the final questionnaire, $72.2 \%$ preferred this experience to explore geometric content over that explored with dynamic geometry software.
\end{abstract}

Key words: Escape Island, toys 2.0, problem solving, Theory of Semiotic Mediation, signs.

\section{INTRODUTION}

A variety of resources can be used in the current digital age to promote meaningful practices inside and outside the classroom. Experiences using both manipulative and digital artifacts, conceived to enhance the building of knowledge show the potential of integration towards the promotion of significative learning (Faggiano, Montone, \& Mariotti, 2018; Maschietto \& Soury-Lavergne, 2013).

To design this learning environments, STEM Education could help teachers think how to combine Science, Technology, Engineering and Mathematics to "pose, ponder, and solve problems that are real world and authentic to our worlds" (MacDonald, Danaia, \& Murphy, 2020, p. 5). Taking advantages of technological artifacts to learn mathematics outside the classroom is a challenge but also an opportunity to promote connections to the real world.

Having as background a study, centered on Mathematics, which aims to investigate the influence of collaborative programming activities on the development of the abilities to solve and pose geometric problems, critical thinking, creativity and collaboration, in this article, we focused on the analysis of the results of one of the tasks that combines problem solving in geometric contexts through the programming of toys 2.0.

In this context, we have set three analysis guiding questions: How do the students think? What kind of signs are they mobilizing? What difficulties do they manifest?

\section{TOYS 2.0}

Originating in Turtle geometry - "computational style of geometry" (Papert. S, 1980, p. 55), several devices are currently available on the market (Figure 1), often called robots that allow the creation of learning environments that combine solving geometric problems through graphic or tangible programming (Figure 1).
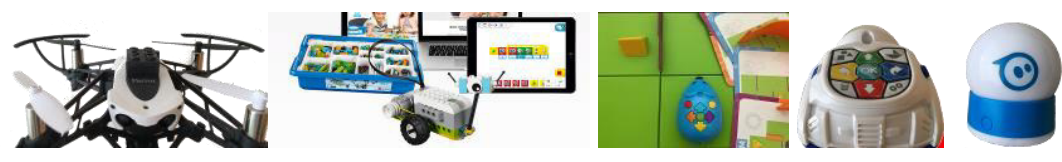

Figure 1: Parrot Mambo, Lego Wedo 2.0, Robot Mouse, Mind Designer Robot, Sphero 2.0.

Cunha, E., Cabrita, I. \& Fonseca, L. (2020). Get out Inside: Programming Toys 2.0 to Escape the Island. In M. Ludwig, S. Jablonski, A. Caldeira, \& A. Moura (Eds.), Research on Outdoor STEM Education in the digiTal Age. Proceedings of the ROSETA Online Conference in June 2020 (pp. 87-94). Münster: WTM. https://doi.org/10.37626/GA9783959871440.0.11 
But what is a robot? Simon (2017) tried to define it so he asked some of the area leading researchers. For Anca Dragan, from the University of Berkeley, "a robot is a physically embodied artificially intelligent agent that can take actions that have effects on the physical world," (Simon, 2017, para. 5). This definition is corroborated by Kate Darling, from the MIT Media Lab. while emphasizing that there is no good universal definition, she considers that a robot "would probably be a physical machine that's usually programmable by a computer that can perform tasks autonomously or automatically by itself" (Simon, 2017, para. 6). Therefore, the incorporation of artificial intelligence is evident. A drone will only be considered a robot if it is autonomous if it is not directly controlled by user actions.

Being a robot cannot be the common property of all devices presented in Figure 1, because, for example, a drone may or may not be, depending on its usage. So, what is the common property? They are 'toys'! 'Toys 2.0.' for bringing an upgrade over traditional toys: the possibility of being programmed.

\section{THEORY OF SEMIOTIC MEDIATION}

Inspired by a Vygotskian approach, shared signs are generated within the social use of artifacts to the accomplishment of a task, involving mediator and mediates. Learning effectiveness depends on the analysis of the artifact's semiotic potential. From this analysis a double semiotic link is established:

on the one hand, personal meanings are related to the use of the artifact, in particular in relation to the aim of accomplishing the task; on the other hand, mathematical meanings may be related to the artifact and its use. (Bussi \& Mariotti, 2008, p. 754)

According to Bussi and Mariotti (2008) we have three categories of signs: artefact signs, pivot signs and mathematical signs. The artefact signs refer to the use of the artefact to carry out the task; the mathematical signs are those that emerge from the mathematical context; and Pivot signs are those which promote the passage between artifact and the mathematical context.

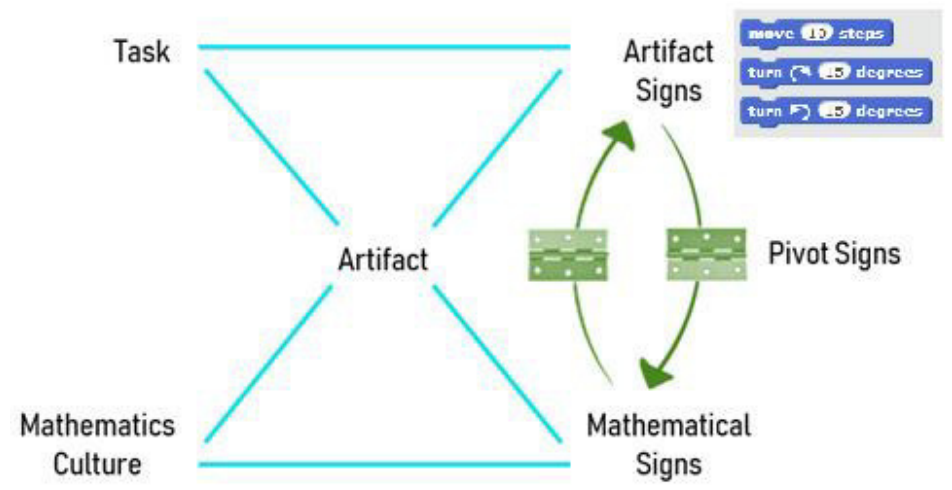

Figure 2: Artefacts and signs in programming tasks, adapted from Bussi and Mariotti (2008, p. 757).

However, when the tasks involve programming toys 2.0, we must consider we are moving from mathematical signs to the artefact signs since programming requires previous mobilization of mathematical signs (from the student's mathematical culture which may or may not be valid). When the toys are tested, new signs are produced: pivot signs, emerging from reflecting about the mistakes made, and new mathematical signs. We can move from artifact signs to mathematical signs and vice versa (Cunha, Cabrita, \& Fonseca, 2019). For 
this reason, the original scheme of Bussi and Mariotti (2008, p. 757) was adapted by placing the arrows in both directions (Figure 2).

\section{METHOD}

In 2018-2019, this study involved 41 pre-service teachers of two 3rd year classes of Technologies in Mathematics Education of the Basic Education course at Viana do Castelo Higher Education School. The Escape Island was part of a series of original tasks and experiences (Figure 3) using Toys 2.0 which were organized in a schedule set to ensure that all students would go through every tasks and experiences.

Each group filmed and photographed each experience to use in the collaborative reflection of each task.

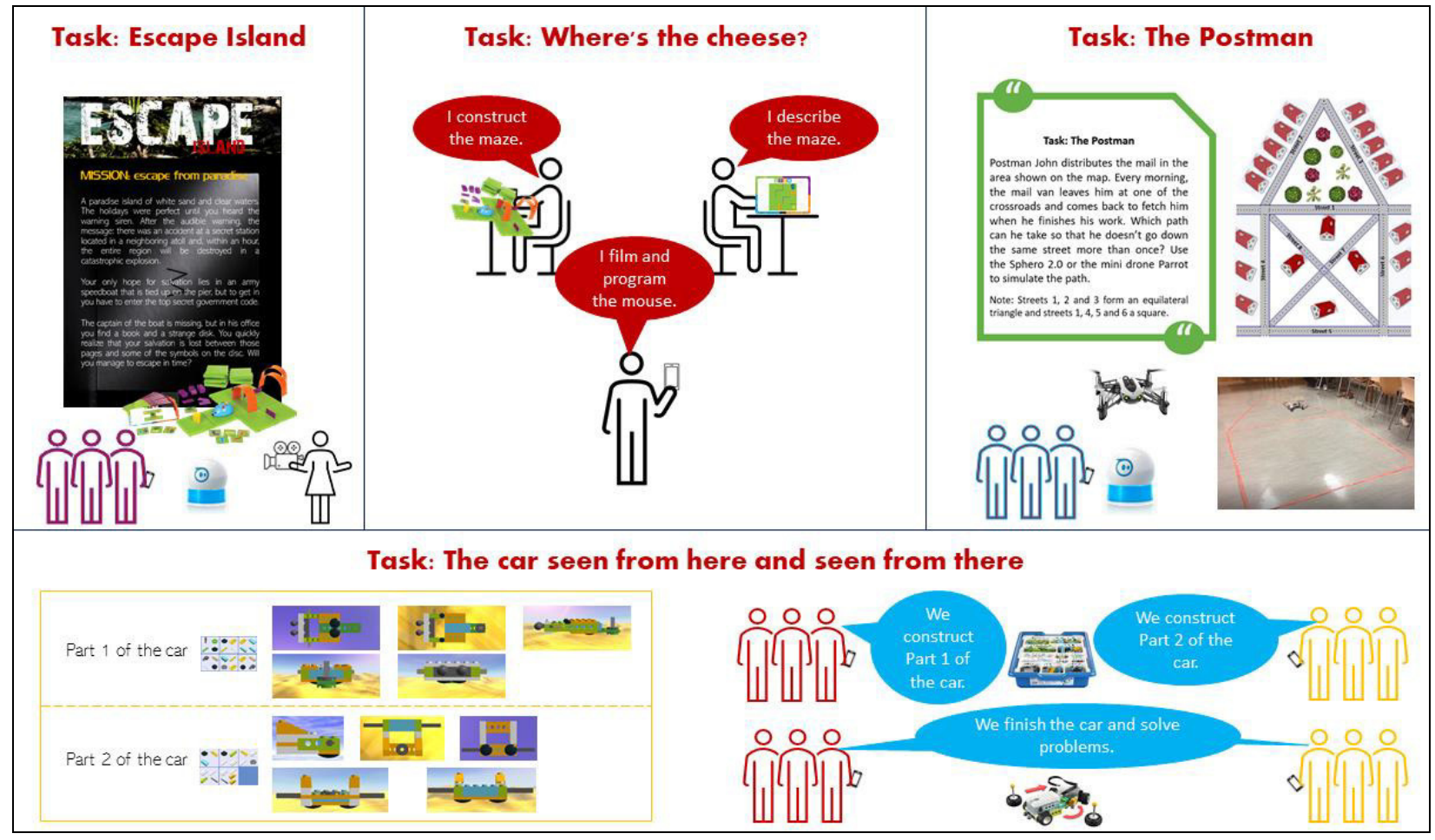

Figure 3: Set of tasks that took place simultaneously.

In this article the analysis will be focused on the task Escape Island. So, in another room, which we identified as the captain's office, in groups of three or four, students had one hour to find a code which would allow them to "escape" an island. To find it, they had to discover hidden clues and solve mysterious puzzles, filled with logical and mathematical problems.

To engage students, the story was presented through the Book of Rules, shown in Figure 4. After that, the students went into the captain's office and found a computer, a tablet, a decoder disk and an emergency manual (Figure 5).

When reading the first page of the Emergency Manual (Figure 6), students realized that unveiling the first clue was related to page 2 of the manual (Figure 7). The symbol at the top of this page was also on the computer at the captain's desk. When clicked, the message showed "Which is the code?" (Figure 8). 


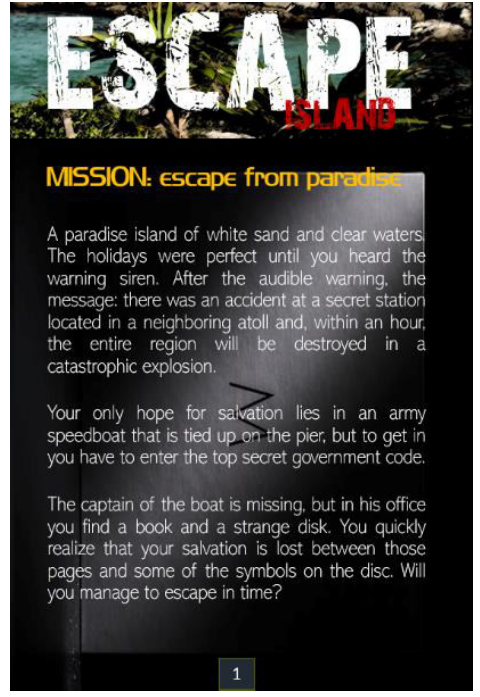

Figure 4: Book rules.

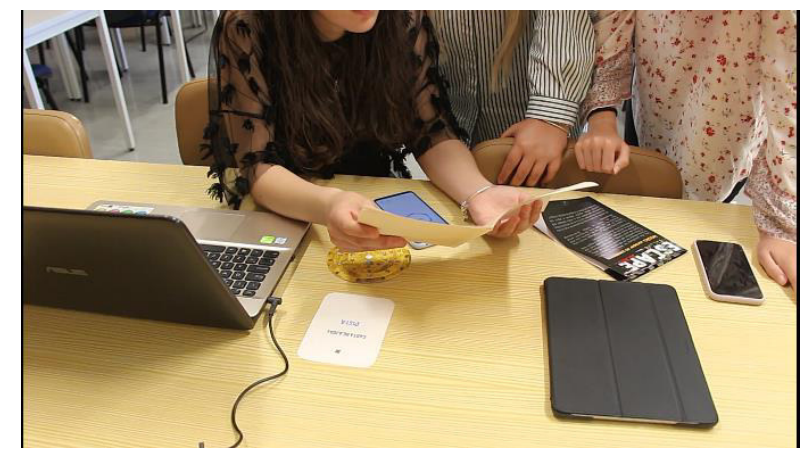

Figure 5: Captain's office.

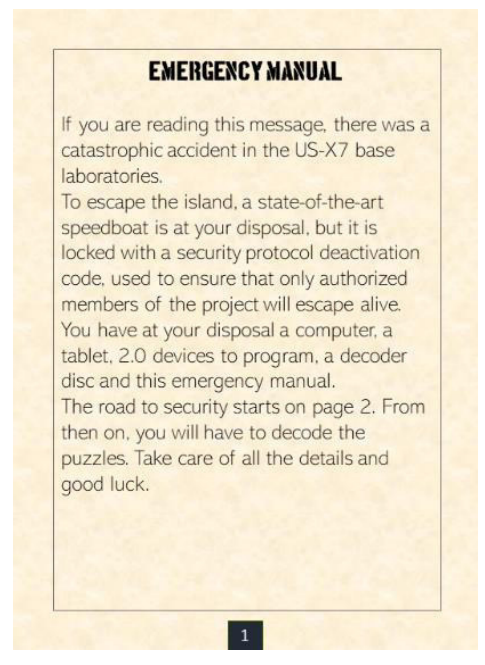

Figure 6: Page 1 of the manual.

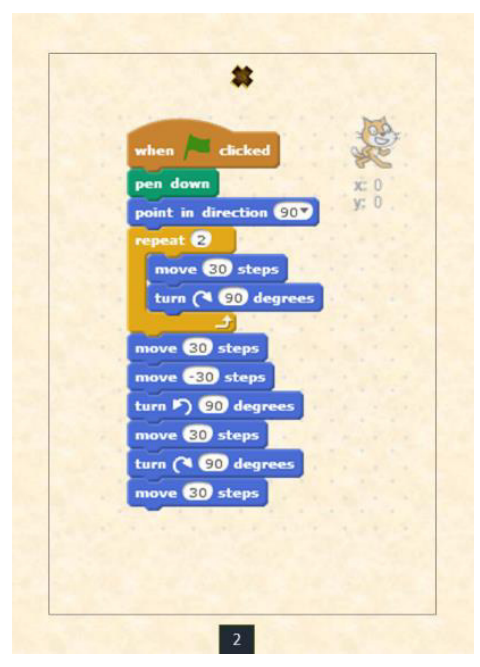

Figure 7: Page 2 of the manual.

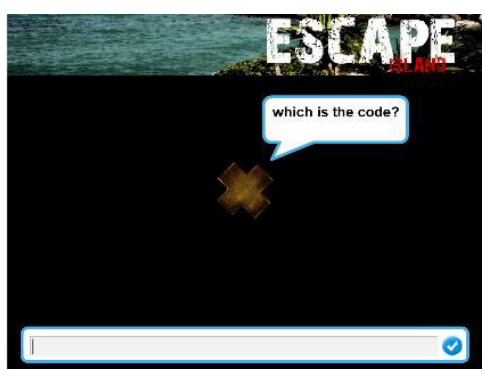

Figure 8: Captain's computer.

The link between clues required students to, without using Scratch, interpret what would be drawn by the cat when the code was activated. When inserted into the computer (the number "3"), it delivered the message: "Code accepted. You have found the first digit required to open the suitcase. Examine the Enigma under the computer".

Enigma A (Figure 9), which was hidden under the computer, gave two clues to discover a three-digit code, required to open the secret equipment bag. The code's hundreds was number 3 and the tens digit would only be available if they removed the blue ball from the model (Figure 10) by programming the Sphero 2.0 (they had to program and use Sphero since all the balls on the model were trapped and would "explode" if touched by something else). To perform this task they had an iPad, which had the Tynker app installed, and some critical information on page 6 of the manual giving them two important clues (Figure 11): the data that the garden on the model was shaped like an isosceles triangle; and the measure of one of its angles.

When the blue ball was pushed out of the model, students could open it. They found the number 2 and the Enigma B card (Figure 12). They had to follow the cat while it moved according to the Scratch code found on page 5 of the manual (Figure 13), to discover three 
symbols which were to be inserted on the decoder disk (Figure 14), recycled from "Exit: The Game - The Forgotten Island (Brand \& Brand, 2017) (note that only the decoder disk was recycled from this game and that all the tasks of Escape Island are original). Decoding the disk, they found the number 7.

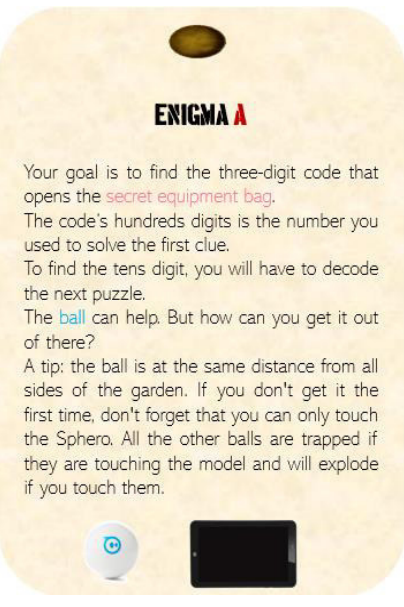

Figure 9: Puzzle A.

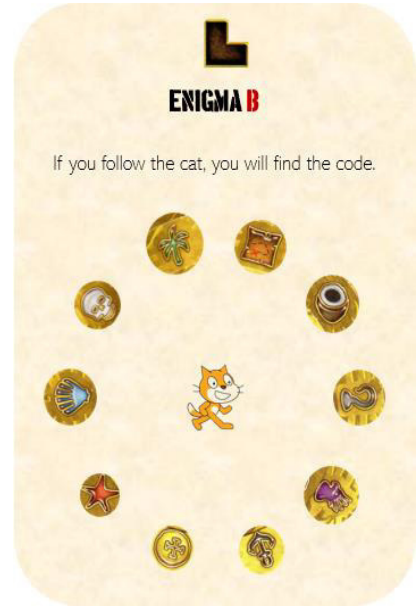

Figure 12: Puzzle B.
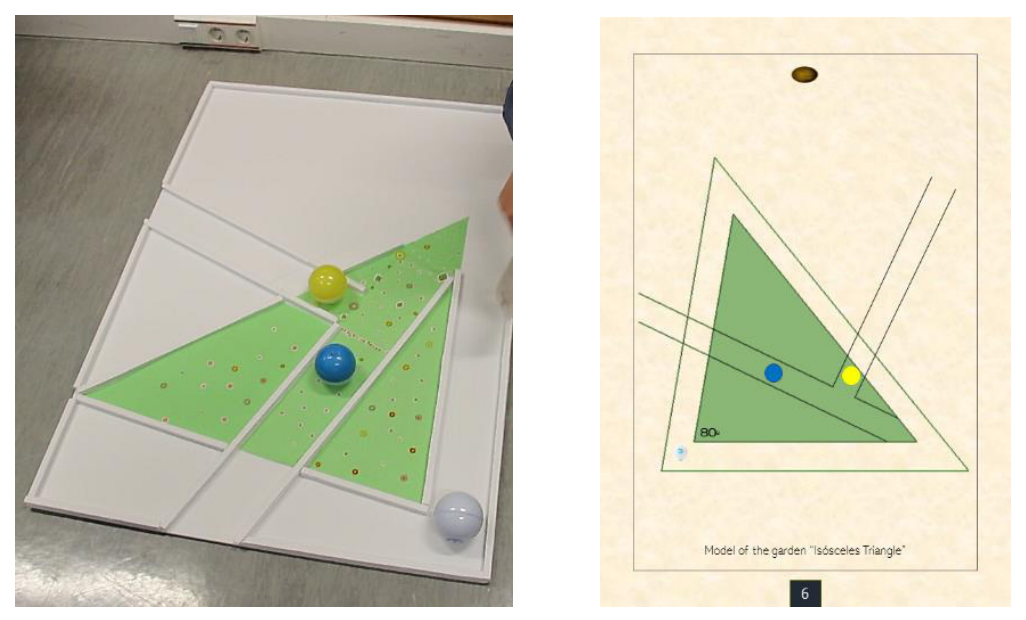

Figure 10: Model of the garden. Figure 11: Page 6 of the manual.

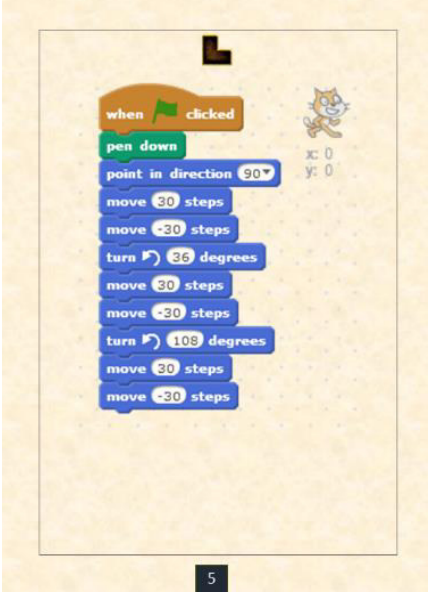

Figure 13: Page 5 of the manual.

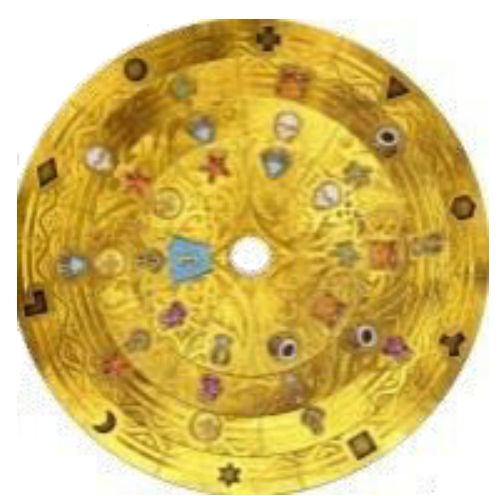

Figure 14: Decoder disk.

So, the code 327 allowed them to open the secret equipment bag. Inside the bag, was the Enigma C card (Figure 15), the robot mouse kit and three of the decoder disk's symbols.

After reading the card, the students had to search the classroom to find the black box shown in Figure 16 (it had the same symbol as the Enigma C card). The box had two holes and they had to use the phone's flashlight to illuminate the interior. Then, one of the members of the group observed what was inside (Figure 17) and gave instructions so that his colleagues could use the robot mouse kit parts to build the maze on the classroom floor. The student who looked inside the box was also able to program the path the mouse should run and he knew where to place it on the maze. Knowing where the mouse would be placed was crucial because they could then use its position as reference to interpret the coordinates associated to the decoders disk symbols (these coordinates were shown on page 4 of the manual). They could then place the symbols on the maze and the order by which they were touched by the mouse (when activated), gave them the code to insert on the decoder disk. The coded resulted on "18", which was the number needed to complete 
the blank spaces on page 3 of the manual. Therefore, "318" was the code that unlocked the escape launch.

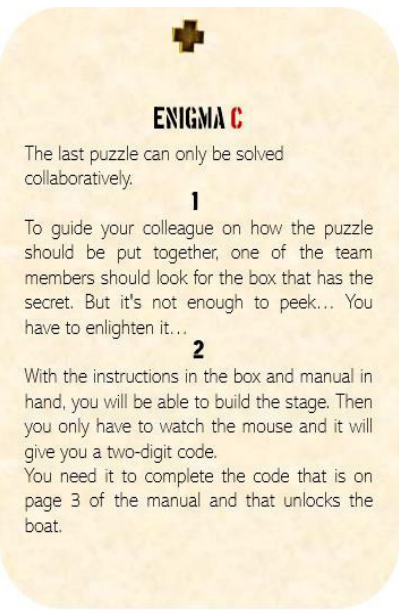

Figure 15: Puzzle C.

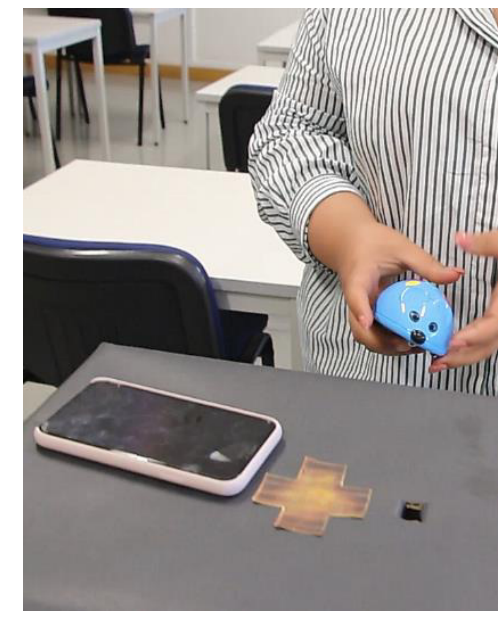

Figure 16: Box that has the secret. Figure 17: Secret inside the box.
Program the shortest path for the mouse to catch the cheese.

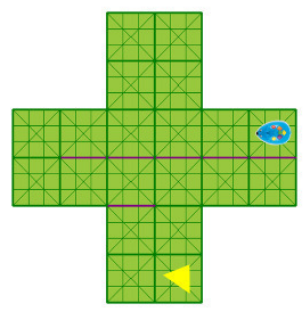

\section{RESULTS}

To solve the first clue, students had to interpret the code shown on Figure 6 and insert it in the computer. Most began by drawing on paper the path which would be drawn by the cat but only one group did it successfully. The remaining groups drew figures as show on Figure 18 and had to try to simulate the cat's path (impersonating it and performing its moves). This experience showed us that all students started from the digital artefact signs Scratch - and all of them could interpret what they meant. But, when they went on to the drawing, although the correct usage of the mathematical signs was obvious (for instance, what " $90^{\circ}$ angle" meant), they quickly lost track of where the pencil was pointing to, leading to several mistakes. Only when recurring to the pivot sign impersonation, using their own body, they could represent the symbol "three".
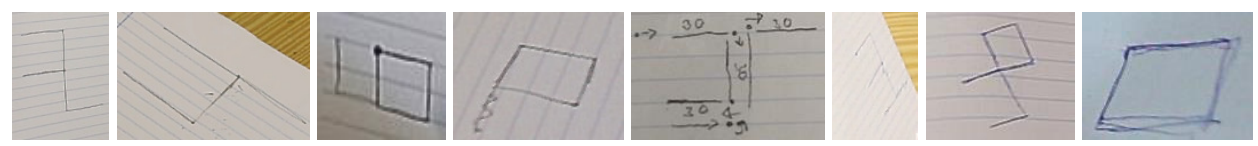

Figure 18: First try examples.

The next enigma demanded that the students programmed Sphero 2.0 to push the blue ball out of the maze. To do it they could use the Tynker app.

To solve this puzzle, they had to mobilize mathematical signs so that they could set a strategy, then move to mobilizing artefact signs and test it. Only in a second phase did most of the groups realize that they were rotating the exterior angle of the triangle and that the blue ball was at the incenter of the triangle. The angle to rotate was 155 degrees, being $(180-25)$. We could see the mobilization of pivot signs, as gestures, among the passage from mathematical signs to the artefact signs, in a cycle of experiences which ended when the ball was finally removed from the garden model.

When they opened the ball, they found "number 2" and the Enigma B card. In this puzzle, they had to follow the cat by using the code available on page 5 of the manual. The interaction was like the one registered on the first experience. The students started from 
the artefact and used their fingers to follow the symbols (pivot sign and mathematical sign). Most solved this problem by estimation, failing to recognize that the symbols were all at the same distance from the cat, so each of the angles in the centre was 36 degrees. For example, when they had to rotate 108 degrees, some groups placed their fingers perpendicularly to show what was the next symbol to place on the decoder disk. One mistake that continued to occur was the fact that they had forgotten where the cat was looking when trying to find the next symbol.

When they opened the suitcase, they found Enigma C. First, they had to search the classroom until they found a box with two holes. One of the holes had to be enlightened by one's cell phone flashlight so that they could look through the remaining hole and see what was inside. Within was the picture of a maze shaped like a cross which was to be reproduced on the classrooms floor. To do so, the student who was looking inside the box had to guide his colleagues on how to assemble the maze correctly. Communication and collaboration were two of the skills mostly highlighted during this task. It was interesting to see that the students frequently used pivot signs, as "pointing", hoping their colleagues would understand what they were trying to say. The information was not always accurate - mathematical signs - which difficulted the assembly of the maze. In the end, the decoding disk symbols were to be placed on the maze using as reference the coordinates were the mouse was to be placed. Some groups had lots of trouble to perform this task.

According to Bussi and Mariotti (2008, p. 753) "gestures, drawings, or words may be the different semiotic means used to produce these signs, the production of which may be spontaneous or explicitly required by the task itself.". However, we should note that, in the programing tasks, we observed gestures, drawings or words being used to mediate the passage from the artifact sign to the mathematical sign but also to mediate the passage from the mathematical sign to the artifact sign (in a trial and error cycle which took place until the correct answer was found).

In this curricular unit's final quiz, the students were questioned on which was the classroom experience they thought had a bigger impact on acquiring concepts and mobilizing strategies to solve problems (between guided concept exploration on a dynamic geometry software and the Escape Island). 18 students answered this question: 13 (72,2\%) stated it was the Escape Island; $3(16,6 \%)$ chose the guided concept exploration on a dynamic geometry software; and $2(11,1 \%)$ did not give a conclusive answer. To justify their answer, 6 focused the ludic, challenging or gaming side of it; 1 the motivational side of it; 3 the importance of wondering about which strategy to use; 1 the mobilization of skills you don't normally use; and 5 focused the remembering/mobilizing/deepening of mathematical concepts. We highlight the answer given by a student which embodies several mentioned aspects and shows the impact this task had to her in the mediation process:

SA: Without a doubt, Escape Island had more impact on me. First, because it was something totally different from everything we had ever done, not for the toys, but for all the context and mystery that hung in the room. Afterwards, all challenges required us to mobilize knowledge that we had acquired in previous classes. In Sphero's challenge, it was even a little shameful to take so long to reach the solution and realize that the ball was in the incenter, because my group no longer remembered what the incenter was. But the mistakes we made and the wrong things we said will make sure I will never forget that the incenter is the intersection point of the bisectors. 


\section{CONCLUSION}

The Escape Island is one of the tasks of a study that intends to analyse the influence of programming activities to develop skills such as problem solving, critical thinking, creativity and collaboration. However, in this paper, we focus only on one of the tasks, the Escape Island challenge, analyzing how students thought, the signs they mobilized, the difficulties which emerged and the students' opinions about the experience.

On challenges which began with artifact signs, namely programming sings, the students were able to correctly interpret the mathematical sign associated with each command. However, when they used a pencil to draw the path set by the commands, they quickly lost track and most of the groups had to use the pivot sign impersonation, using their own body to follow the instructions correctly (without losing track). On the puzzle which demanded they removed a ball from the model, the students began by activating mathematical signs but, before transforming them into programming sings, most of them used their own bodies to simulate the movement they expected the ball would make. After this experimentation, they tried programming and, in a cycle of several attempts, they confirmed if the initial conceptions were correct, mobilizing new knowledge and reflecting critically on their mathematical culture. It should also be noted that in the last challenge when communicating the steps to build the maze, students tended to point instead of communicating using mathematical signs.

Finally, the students presented evidence that the experience was significant and remarkable for their learning.

\section{References}

Brand, I., \& Brand, M. (2017). Exit: the game - the forgotten island. Devir.

Bussi, M. G. B., \& Mariotti, M. A. (2008). Semiotic mediation in the mathematics classroom Artifacts and signs after a Vygotskian perspective. In L. English (Ed.), Handbook of International Research in Mathematics Education (pp. 746-783). Routledge. Retrieved from http://www.cfem.asso.fr/actualites/archives/bartolini-mariotti_handbook

Cunha, E., Cabrita, I., \& Fonseca, L. (2019). Graphic programming artefacts in the development of geometric skills. In CIEAEM71 (pp. 164-170). Braga, Portugal.

Faggiano, E., Montone, A., \& Mariotti, M. A. (2018). Synergy between manipulative and digital artefacts: a teaching experiment on axial symmetry at primary school. International Journal of Mathematical Education in Science and Technology, 49(8), 1165-1180. https://doi.org/10.1080/0020739X.2018.1449908

MacDonald, A., Danaia, L., \& Murphy, S. (Eds.). (2020). STEM Education Across the Learning Continuum: Early Childhood to Senior Secondary. Singapore: Springer Singapore. https://doi.org/10.1007/978-981-15-2821-7

Maschietto, M., \& Soury-Lavergne, S. (2013). Designing a duo of material and digital artifacts: the pascaline and Cabri Elem e-books in primary school mathematics. ZDM, 45(7), 959-971. https://doi.org/10.1007/s11858-013-0533-3

Papert. S. (1980). Mindstorms Childern computer and powerful ideas. New York: Basic Books, Inc. Retrieved from http://dl.acm.org/citation.cfm?id=1095592

Simon, M. (2017). What Is a Robot? | WIRED. Retrieved October 29, 2018, from https://www.wired.com/story/what-is-a-robot/ 